\title{
Evaluation of doxorubicin-eluting bead transcatheter arterial chemoembolization combined with endovascular brachytherapy for hepatocellular carcinoma with main portal vein tumor thrombus
}

\section{Zhiyuan Wu}

Shanghai Jiao Tong University Medical School Affiliated Ruijin Hospital Ju Gong

Ruijin Hospital Luwan Branch $₫$ Shanghai Jiao Tong University of Medicine

Wei Huang

Shanghai Jiao Tong University Medical School Affiliated Ruijin Hospital

Qingbing Wang

Shanghai Jiao Tong University Medical School Affiliated Ruijin Hospital

\section{Ziyin Wang}

Shanghai Jiao Tong University Medical School Affiliated Ruijin Hospital

Qin Liu

Shanghai Jiao Tong University Medical School Affiliated Ruijin Hospital

Jingjing Liu

Shanghai Jiao Tong University Medical School Affiliated Ruijin Hospital

Junwei Gu

Shanghai Jiao Tong University Medical School Affiliated Ruijin Hospital

\section{Xiaoyi Ding}

Shanghai Jiao Tong University Medical School Affiliated Ruijin Hospital

\section{Zhongmin Wang ( $\nabla$ wzm0722@hotmail.com)}

Affiliated Ruijin Hospital, Shanghai Jiao Tong University School of Medicine, https://orcid.org/00000001-7994-4538

\section{Research article}

Keywords: Carcinoma, hepatocellular, portal vein, stents, endovascular brachytherapy, chemoembolization, doxorubicin-eluting beads.

Posted Date: September 28th, 2020

DOl: https://doi.org/10.21203/rs.3.rs-52846/v2 
License: (c) (i) This work is licensed under a Creative Commons Attribution 4.0 International License. Read Full License 


\section{Abstract}

Background The goal of this study was to compare the clinical results of conventional transcatheter arterial chemoembolization (C-TACE) and doxorubicin-eluting bead transcatheter arterial chemoembolization (D-TACE) combined with endovascular stent implantation with an iodine-125 seed strand in hepatocellular carcinoma (HCC) patients with main portal vein tumor thrombus (MPVTT).

Methods This study was a prospective controlled study with follow-up dates spanning from Mar 2015 to Feb 2020. Patients with both HCC and MPVTT were randomly divided into two groups. Portal vein stents with iodine-125 seed strands were implanted first; then, C-TACE or D-TACE was administered to all patients. Objective response rates were assessed. The time to disease progression and survival rate were compared between the two groups.

Results A total of 26 patients were enrolled, with 13 in each group. During follow-up, the portal stent patency times were $112.3 \pm 98.2$ days in the C-TACE group and 101.7 \pm 90.4 days in the D-TACE group. The time to disease progression was 42 days in the C-TACE group and 120 days in the D-TACE group $(p=0.03)$. The overall survival time from the first intervention procedure was 216 days in the C-TACE group and 239 days in the D-TACE group $(p=0.047)$. The D-TACE group was superior to the C-TACE group in terms of progression-free survival (PFS) and overall survival (OS) times.

Conclusion Endovascular implantation of a stent with an iodine- 125 seed strand combined with TACE is safe and effective in HCC patients with MPVTT. Compared to C-TACE, D-TACE achieves more benefits regarding PFS and OS.

Trial registration This study was a cohort study, no health-related interventions to evaluate the effects on health outcomes. This study wasn't a clinical trial.

\section{Background}

Hepatocellular carcinoma (HCC) is a common malignant tumor. HCC patients with main portal vein tumor thrombus (MPVTT) often miss the opportunity for transcatheter arterial chemoembolization (TACE), a preferred nonsurgical option for liver cancer, because MPVTT is a relative contraindication for TACE. Previous studies have demonstrated that this type of patient may be able to have TACE through stent implantation into the portal vein with thrombosis to recover portal blood flow [1]. The implantation of a radioactive seedling tent into the portal vein stent combined with TACE and MPVTT increases the stent patency rate and survival time [2,3]. Conventional TACE (C-TACE) embolic materials are always based on multi-iodized oil. A recent study reported the application of new embolic material loaded with doxorubicin class drug-eluting beads (DEB). Preliminary results show that the efficacy of DEB-TACE (D-TACE) in HCC is good and has low toxicity [4]. However, whether D-TACE has greater survival benefits is still controversial [5]. A DEB product, DC Bead ${ }^{\mathrm{TM}}$ (Biocompatibles UK Ltd.), has been officially approved by the China Food and Drug Administration and launched in China. However, few studies have examined the effects of radioactive seed stent implantation in a portal vein stent combined with D-TACE. 
This preliminary study aimed to investigate the feasibility and safety of implanting a radioactive seed stent in a portal vein stent combined with TACE in HCC patients with MPVTT, and it compared the clinical effects of combined therapies with C-TACE or D-TACE.

\section{Methods}

\section{Subjects}

In this prospective controlled study, 26 HCC patients with MPVTT were consecutively enrolled from March ${ }^{1}, 2015$,to August 31, 2019 with subsequent follow-ups until February 29, 2020.This study was approved by the ethics committee of .Ruijin Hospital Affiliated to Shanghai Jiao Tong University School of Medicine.

The included patients met the following conditions: age 18 to 80 years; diagnosis met the pathological or clinical diagnostic criteria of HCC; CT or MRI imaging showed that portal vein thrombosis involved the portal vein trunk and primary branch, but that the contralateral primary branch was not completely occluded; the liver functionstage was Child-Pugh class AB; patients had no extensive extrahepatic metastases; and the Eastern Cooperative Oncology Group (ECOG) score of the patient was 0-2. Due to economic constraints or concerns about the side effects of targeted drugs, these patients were not able to combine targeted drugs at the same time. All patients signed informed consent forms for this study.

Patients were excluded for the following reasons: the liver function stage was Child-Pugh class C; the patient had other serious diseases and could not complete treatment; or the patient had bleeding tendency with elongated coagulation time.

\section{Intervention procedures}

All patients underwent radioactive seeding in the portal vein immediately followed by C-TACE or D-TACE. First, under the guidance of ultrasound, the portal vein branch of the uninvolved liver lobe was percutaneously punctured and a vascular sheath was inserted. Second, angiography was performed over the main portal vein stenosis segment with a $4 \mathrm{~F}$ pigtail catheter (Cordis, USA), and the pressure was measured. The diameter and length of the stent as well as the number of required iodine-125 $\left({ }^{125} \mathrm{I}\right)$ seeds were based on the stenosis segment length. The stents should extend $1 \mathrm{~cm}$ beyond each end of the tumor thrombus. The number of ${ }^{125} \mathrm{I}$ seeds $(0.6 \mathrm{mCi} /$ tablets, Shanghai Xinke Pharmaceutical Co., Ltd.) were calculated using the formula [stenosis length $(\mathrm{mm}) / 4.5+2$ ] to ensure that the radiation range of implanted ${ }^{125}$ I seeds completely covered the portal vein thrombosis segment. The required ${ }^{125}$ I seedlings were encapsulated in a $3 \mathrm{~F}$ sterile sheath and developed into a seed strand. Third, the stents and the ${ }^{125}$ I seed strips were placed into the portal vein vessels of the stenosis segments under the guidance of the Xray perspective. Fourth, portal vein angiography was performed with the pigtail catheter; the pressure at this time was measured again. The liver puncture channel was blocked with a $5 \mathrm{~mm} \times 5 \mathrm{~cm}$ coil (Cook Company, USA). 
In the C-TACE group, the lipiodol dosage was based on lesion size. A tumor with a diameter of $1 \mathrm{~cm}$ corresponded to $1 \mathrm{ml}$ of lipiodol with a maximum dose of $20 \mathrm{ml}$. Epirubicin (40 mg, Pharmorubicin, Pfizer) was mixed with the lipiodol, forming an emulsion. In the D-TACE group, the doxorubicin-eluting (DC) bead diameters were $300 \mu \mathrm{m}$ to $500 \mu \mathrm{m}$. One to two bottles were used based on lesion size, each containing $40 \mathrm{mg}$ epirubicin. After combining the epirubicin with DC beads, a nonionic contrast agent iopamidol injection $(370 \mathrm{mg} \mathrm{l} / \mathrm{mL}$ ) was mixed at a 1:1 proportion. TACE was performed using the femoral artery approach. The abovementioned embolic agents were slowly injected into the tumor-feeding artery for embolization after superselective catheterization. Gelatin sponges were added to strengthen the embolism.

Patients received liver protection treatment with symptomatic and supportive treatment for 7 to 8 days after interventional procedures. Blood tests, liver renal function tests, and electrolytes were measured at 3 days, 7 days, 14 days and 30 days after the procedures. Complications were recorded and treated accordingly. Follow-up was performed every 3 months after the initial treatment. The clinical results mainly includes the changes of liver function, complications, the time to disease progression (estimated using enhanced abdominal CT or abdominal MRI) and survival rate. Objective response rates were assessed, and TACE treatments were performed as needed. The patency of the portal stent was compared between the two groups. The median progression-free survival (PFS) and overall survival (OS) times were assessed after long-term follow-up.

\section{Statistical analyses}

The statistical analyses were performed using SPSS statistical software, version 23 (IBM, Armonk, NY, USA). A paired t-test was used to compare the changes in portal vein pressure before and after stent implantation. The Mann-Whitney test was used to compare the liver function and stent patency of the two groups. Progression-free survival and overall survival were analyzed with Kaplan-Meier and log-rank tests. All data are expressed as the mean \pm SEM (standard error of the mean) of $n$ independent measurements. GraphPad Prism 7 software (GraphPad, San Diego, CA, USA) was used to plot the graphs. A value of $P<0.05$ was considered as statistically significant.

\section{Results}

Twenty-six patients (aged 40-78 years) were included in the analysis. The median age of the C-TACE group ( 9 men and 4 women) was 54.5 years; in the D-TACE group ( 11 men and 2 women), the median age was 56 years. Based on BCLC staging criteria, the all the patients were in stage $C$. Based on the ChildPugh classification standard of liver function, in the C-TACE group, 9 cases were Child-Pugh class A, the other 4 cases were class $B$, and the mean Child-Pugh score was 6.15 \pm 0.90 . In the D-TACE group, 11 cases were Child-Pugh class $A$, the other 2 cases were class $B$, and the mean Child-Pugh score was $5.77 \pm 0.73$. No significant differences were found between the two groups (Mann-Whitney $U=64.5, p=0.31$ ). All patients had HCC lesions. In the C-TACE group, there were 4 cases in the left liver and 9 cases in the right liver. In the D-TACE group, there was one case in the left liver and all other cases were in the right liver. All 
the patients had tumor-side portal vein branch thrombus and MPVTT. Among these, 6 cases were accompanied by contralateral first level portal vein branch tumor thrombi in the C-TACE group and 4 cases were accompanied by contralateral first level portal vein branch tumor thrombi in the D-TACE group.There was no significant difference in post embolization syndrome between two groups.

All the patients successfully underwent stent implantation of a radioactive seed into the portal vein followed by TACE treatment. In the C-TACE group (Figure 1), 13 stents were implanted (diameter: 8-14 $\mathrm{mm}$, length: $60-90 \mathrm{~mm}$ ), $13^{125}$ I radioactive seeds were used (a total of $204^{125}$ I seeds, with an average of 16 per strip), and $10.6 \mathrm{ml}$ of lipiodol was used in each case on average. Fifty milligrams of epirubicin were mixed with lipiodol for each case, and 15 boxes of gelatin sponges were used to enhance embolization. In the D-TACE group (Figure 2), 13 stents were implanted (diameter: 8-14 mm, length: 40-94 $\mathrm{mm}$ ), and $13^{125} \mathrm{I}$ radioactive seeds were used (a total of $181^{125} \mathrm{I}$ seeds, with an average of 14 per stripe); 18 bottles of DC beads were used; and five boxes of gelatin sponges were used to enhance embolization. Each bottle of DC beads contained a mixture of $40 \mathrm{mg}$ of epirubicin.

Intraoperative angiography of the portal vein showed that the portal vein length of the tumor thrombus was 25.3 to $95.2 \mathrm{~mm}$ (average: $46.6 \pm 19.1 \mathrm{~mm}$ ) in the C-TACE group and 17.6 to $65.7 \mathrm{~mm}$ (average: $38.8 \pm 16.5 \mathrm{~mm}$ ) in the D-TACE group. In the C-TACE group, the average pressure of the distal main portal vein was $28.3 \pm 11.2 \mathrm{cmH}_{2} \mathrm{O}$ and $23.6 \pm 10.2 \mathrm{cmH} 2 \mathrm{O}$ before and after stent implantation, respectively, a decrease of $4.6 \pm 3.0 \mathrm{cmH}_{2} \mathrm{O}$, but the difference before and after stent implantation was not statistically significant $(\mathrm{U}=74.5, \mathrm{P}=0.62)$. In the $\mathrm{D}$-TACE group, the average pressures were $25.2 \pm 12.3 \mathrm{cmH}_{2} \mathrm{O}$ and $20.2 \pm 11.7 \mathrm{~cm} \mathrm{H}_{2} \mathrm{O}$, respectively, a decrease of $5.0 \pm 4.4 \mathrm{cmH} 2 \mathrm{O}$, which was also not statistically significant $(U=62.5, P=0.27)$. The difference in the magnitude of pressure decrease between the two groups was not statistically significant $(U=74.5, P=0.62)$.

The alanine aminotransferase (ALT), aspartate aminotransferase (AST) and serum total bilirubin (TBil) levels significantly increased at 3 days and 7 days after the procedures but returned to preoperative levels after 30 days (Table 1). There were no significant differences in Child-Pugh scores between the two groups before the procedures $(p=0.47)$ or at 3 days $(p=0.77), 7$ days $(p=0.66), 14$ days $(p=0.47)$, and 30 days $(p=0.56)$ after the procedures (Figure 3$)$.

Table 1. Liver function parameters before and 3 days, 7 days, 14 days, 30 days after interventional procedures 


\begin{tabular}{cccccc}
\hline & & ALT $(\mathrm{IU} / \mathrm{L})$ & AST $(\mathrm{IU} / \mathrm{L})$ & TBil $(\mu \mathrm{mol} / \mathrm{L})$ & Child-Pugh score \\
\hline C-TACE group & pre & $48.6 \pm 28.9$ & $84.6 \pm 43.7$ & $30.6 \pm 19.1$ & $5.7 \pm 1.3$ \\
& 3d & $100.3 \pm 151.0$ & $210.0 \pm 356.5$ & $37.6 \pm 19.9$ & $6.8 \pm 1.9$ \\
& 7d & $132.6 \pm 154.7$ & $176.1 \pm 135.0$ & $48.5 \pm 42.9$ & $6.8 \pm 1.0$ \\
& 14d & $90.7 \pm 29.9$ & $106.0 \pm 58.0$ & $103.6 \pm 122.9$ & $6.8 \pm 0.7$ \\
& 30d & $34.5 \pm 54.7$ & $108.8 \pm 56.8$ & $53.3 \pm 94.2$ & $6.3 \pm 0.9$ \\
\hline D-TACE group & pre & $53.8 \pm 42.8$ & $93.2 \pm 54.5$ & $21.0 \pm 7.0$ & $5.3 \pm 1.3$ \\
& 3d & $451.3 \pm 462.8$ & $590.3 \pm 428.9$ & $47.9 \pm 26.9$ & $6.7 \pm 2.0$ \\
& 7d & $200.8 \pm 222.6$ & $116.2 \pm 67.6$ & $51.6 \pm 27.9$ & $6.6 \pm 1.3$ \\
& 14d & $121.7 \pm 75.7$ & $127.0 \pm 121.4$ & $123.1 \pm 165.2$ & $6.5 \pm 1.3$ \\
& 30d & $47.7 \pm 45.1$ & $117.5 \pm 145.8$ & $51.5 \pm 77.7$ & $6.0 \pm 1.2$ \\
\hline
\end{tabular}

ALT: alanine aminotransferase; AST: aspartate aminotransferase; TBil: serum total bilirubin

No patient in either group had serious complications such as puncture bleeding, abdominal bleeding, tumor rupture, gastrointestinal bleeding, liver abscess, or bile aneurysm. Postoperative adverse reactions included postembolization syndrome, nausea, pain, fever and fatigue, all of which significantly improved after symptomatic treatment. Two patients with myocardial damage had chest discomfort and pain within 24 hours after the procedures in the D-TACE group treated with $80 \mathrm{mg}$ (mixed with 2 bottles of DC beads) and $40 \mathrm{mg}$ epirubicin (mixed with 1 bottle of DC beads), respectively. No abnormal electrocardiogram findings were observed, but the serum levels of AST, lactate dehydrogenase (LDH), and $\mathrm{N}$-terminal pro B-type natriuretic peptide (NT-proBNP) within 24 hours after the procedures had transient increases. After oxygen therapy, sublingual nitroglycerin and other treatments, the indicators of myocardial damage gradually decreased after 3 days.

During the follow-up, the stent patency time of the two groups was $112.3 \pm 98.2$ days in the C-TACE group and $101.7 \pm 90.4$ days in the D-TACE group, and there was no significant difference between the groups $(U=84, p>0.99)$ (Figure 4).

According to the mRECIST criteria[6], the increased enhanced tumor tissue volume in CT or MRI images was used as the basis for evaluating disease progression. The median progression-free survival times of the two groups were 42 days and 120 days, respectively-significantly longer in the D-TACE group than in the C-TACE group $(p=0.03$ ) (Figure $5 A)$. By the end of the 5 -year follow-up, 3 patients in the D-TACE group still survived. From the initial diagnosis, the overall survival times were 235 days and 357 days in the two groups $(p=0.02)$ (Figure $5 B)$. From the first interventional procedures, the overall survival times of the two groups were 216 days and 239 days, respectively $(p=0.047)$ (Figure $5 C$ ). The difference was statistically significant: the D-TACE group was superior to the C-TACE group regarding both PFS and OS.

\section{Discussion}


This study demonstrated that endovascular implantation of a stent with a ${ }^{125}$ I seed strand combined with D-TACE is a safe and effective option for managing HCC patients with MPVTT.

Portal vein thrombosis is an important factor in the prognosis of HCC patients with portal vein tumor thrombus; the natural survival of these patients is approximately 4 months [7]. MPVTT blocks the portal vein blood flow in the liver, which may have a significant impact on liver reserve function and increase the risk of gastrointestinal bleeding caused by secondary portal hypertension. Previous studies have reported on the results of combinations of targeted drugs [8], radiation therapy [9], thermal ablation [10] and other methods based on C-TACE in treating HCC with MPVTT. Portal vein revascularization through endovascular stenting can improve liver parenchymal perfusion, relieve sequelae of portal hypertension and lead to the regaining of subsequent liver function [11]. The implantation of a radioactive seeds stent in a portal vein stent with TACE showed greater advantages [2], including increased safety and efficacy as well as a higher stent patency rate. In this study, the mean stent portal vein pressure was $28.3 \pm 11.2$ $\mathrm{cmH}_{2} \mathrm{O}$ in the C-TACE group and $25.2 \pm 12.3 \mathrm{cmH}_{2} \mathrm{O}$ in the D-TACE group, which decreased to $23.6 \pm 10.2$ $\mathrm{cmH} 2 \mathrm{O}$ and $20.2 \pm 11.7 \mathrm{cmH}_{2} \mathrm{O}$, respectively, after the stent implantations, suggesting that implanting portal vein stents may decrease the portal vein pressure and reduce the risk of secondary gastrointestinal bleeding in patients with MPVTT.

Endovascular brachytherapy with ${ }^{125}$ I seeds can be continuously performed and has a long half-life (approximately 60.1 d). Close contact with the tumor tissue under the continuous emission of X-rays and $Y$-rays from the ${ }^{125}$ I seed can destroy the double-stranded DNA of tumor cells and inhibit the growth of tumor thrombi [2]. Local irradiation can also inhibit vascular endothelial proliferation [13] and prolong the duration of stent patency. In this study, an appropriate amount of ${ }^{125} \mathrm{I}$ seeds were packaged into $3 \mathrm{~F}$ sterile sheaths to develop seed strains based on tumor thrombus length (measured by main portal vein angiography); then, the patients were synchronously implanted with a portal vein stent and the stent was expanded. The ${ }^{125}$ I seed strains were fixed to the tumor thrombus site, effectively preventing loss and displacement. Portal vein stent implantations were successfully completed in all the patients and a ${ }^{125}$ seed strain was implanted, suggesting that this combined therapy method is highly feasible.

Percutaneous puncture of the portal vein branch under ultrasound guidance is a relatively safe procedure. Effective image guidance avoids blind punctures, which may damage vessels or bile ducts. Compared with direct implantation of ${ }^{125}$ I seeds into MPVTT, the preinstalled seed strains avoided damage caused by repeated punctures at a fixed site and reduced the risk of ectopic implantation.

Previous studies have shown [14] that compared with C-TACE, patients' tolerance of D-TACE is better, with less-severe liver toxicity and fewer doxorubicin-related side effects. However, no study has reported the combination of radioactive seed stent implantation into the portal vein and the application of D-TACE in the treatment of HCC patients with MPVTT. The present study preliminarily explored the feasibility of this combination by investigating blood tests, coagulation, and liver and kidney function during the perioperative period as well as the safety of this treatment combination. Our results showed that all the 
patients successfully completed the procedures under local anesthesia with good tolerance and no serious complications. Liver function was transiently abnormal but recovered gradually. The recovery of serum Tbil was slower than that of ALT and AST, which may be related to bile duct injury after embolism. The parameters of two patients with myocardial damage gradually decreased 2 to 3 days after oxygen therapy and nitroglycerin sublingual treatments. The epirubicin dosages of the two patients were $80 \mathrm{mg}$ and $40 \mathrm{mg}$, respectively; based on patients' body surface area, these were not overdoses, suggesting the possible cardiotoxicity of D-TACE when loaded with epirubicin or doxorubicin. Therefore, patient heart function needs to be monitored closely and treated promptly. Overall, the combination of the implantation of radioactive seed stents into the portal vein and D-TACE in the treatment of HCC patients with MPVTT is both feasible and safe.

D-TACE has been applied as a treatment for HCC for many years, and a number of clinical trials have demonstrated its safety [15] and effectiveness [4]. A DC Bead ${ }^{\circledR}$ is a drug-loaded microsphere that was approved in China in August 2014. The bead can be loaded with doxorubicin, epirubicin, or irinotecan. After embolism, the drugs can be continuously released with a certain amount of compressibility, which can effectively block the target vessel. One prospective randomized controlled study [14] compared CTACE with D-TACE in the treatment of HCC and found that the complete remission rate, objective response rate and disease control rate in the D-TACE group were higher than those in the C-TACE group ( $27 \%$ vs $22 \%, 52 \%$ vs $44 \%$, and $63 \%$ vs $52 \%$, respectively) but without significant differences. However, the objective response rate in the D-TACE group was significantly higher than that of the C-TACE group among cases with Child-Pugh grade B, ECOG score 1, involvement of two lobes, and relapse.

A randomized controlled study [16] compared the effectiveness of simple microembolization (BB group) and the drug doxorubicin in microsphere embolization (LCB group) in patients with HCC and found that the response rates (RECIST criteria) of the BB group and LCB group at the first revisit were $5.9 \%$ and $6.0 \%$, respectively. The median PFS values of the BB group and LCB group were 6.2 months and 2.8 months, respectively, which were not significantly different. The median overall survival of the BB and LCB groups were 19.6 months and 20.8 months, respectively, which were not significantly different. The embolization effects of ordinary microspheres and drug-loaded adriamycin microspheres on $\mathrm{HCC}$ were not significantly different. Drug-loaded adriamycin microspheres may not be able to improve the effectiveness of liver cancer embolization. The long-term efficacy of D-TACE needs further investigation [17]. Therefore, we followed up these cases for 5 years, a length of time that not only allows assessing the feasibility and safety of the combined treatment method but also objectively evaluating the long-term efficacy of the combined treatment. In our study, the D-TACE group was superior to the C-TACE group regarding both PFS and OS. Compared with other studies, our patients had a relatively late disease course, showing that the combined treatment may have more advantages for patients with more severe disease.

Our study had some limitations. The sample size was relatively small. More cases are needed to reach more reliable results. All these patients were in the BCLC-C stage, and treatment with targeted drugs such as sorafenib may have yielded better results[18]. However, due to economic constraints or concerns about the side effects of targeted drugs, these patients were not able to combine targeted drugs at the same 
time. Follow-up studies are also needed on patients who are being treated with combined targeted drug therapies.

\section{Conclusion}

In conclusion, the combination of the implantation of a radioactive seed stent to the portal vein and DTACE in the treatment of HCC patients with MPVTT is both safe and feasible. This combination therapy may offer increased survival benefits to patients with stage BCLC-C liver cancer.

\section{Abbreviations}

C-TACE\conventional transcatheter arterial chemoembolization

D-TACE囚doxorubicin-eluting bead transcatheter arterial chemoembolization

HCC囚hepatocellular carcinoma

MPVTT 囚main portal vein tumor thrombus

PFS『progression-free survival

OS: overall survival

DEB: drug-eluting beads

ECOG: Eastern Cooperative Oncology Group

\section{Declarations}

\section{Ethics approval and consent to participate[}

This study was approved by the ethics committee of Ruijin Hospital affiliated to Shanghai Jiaotong University of Medicine. The ethics approval number was B2016-106R.A written consent was obtained from each participants.

\section{Consent for publication}

Not applicable

\section{Availability of data and materials!}

The datasets used and/or analysed during the current study are available from the corresponding author on reasonable request

Competing interests $\square$ 
The authors declare that they have no competing interests.

\section{Funding[}

National Natural Science Foundation of China (contract grant number: 81771949); Shanghai Municipal Commission of Health and Family Planning (contract grant number: 201640087) had supported analysis of this study;Shanghai Municipal Key Clinical Specialty(contract grant number: shslczdzk07002, shslczdzk06002) had design of the study and collection;Shanghai KeySpecialty Construction Project (contract grant number: ZK2019A02) had interpretation of data and in writing the manuscript.

\section{Authors' contributions[}

In this study, WZY designed the research and drafted the paper. GJ and HW made substantial contributions on analysis and interpretation of the data. WQB, WZY and LQ conducted research and acquisition data. LJJ made great contributions on statistical analysis. GJW made substantial contributions on administrative, technical, and material support. DXY made critical revision for this manuscript. WZM made great contributions on study supervision, obtained funding and final approval of the version. All authors read and approved the final manuscript.

\section{Acknowledgements [}

Not applicable.

\section{References}

1. Zhang XB, Wang JH, Yan ZP, Qian S, Liu R: Hepatocellular carcinoma invading the main portal vein: treatment with transcatheter arterial chemoembolization and portal vein stenting. Cardiovasc Intervent Radiol 2009, 32(1):52-61.

2. Luo JJ, Zhang ZH, Liu QX, Zhang W, Wang JH, Yan ZP: Endovascular brachytherapy combined with stent placement and TACE for treatment of $\mathrm{HCC}$ with main portal vein tumor thrombus. HEPATOL INT 2016, 10(1):185-195.

3. Wu YF, Wang T, Yue ZD, Zhao HW, Wang L, Fan ZH, He FL, Liu FQ: Stents combined with iodine-125 implantation to treat main portal vein tumor thrombus. World J Gastrointest Oncol 2018, 10(12):496504.

4. Poon RT, Tso WK, Pang RW, Ng KK, Woo R, Tai KS, Fan ST: A phase I/II trial of chemoembolization for hepatocellular carcinoma using a novel intra-arterial drug-eluting bead. Clin Gastroenterol Hepatol 2007, 5(9):1100-1108.

5. Gomes AS, Monteleone PA, Sayre JW, Finn RS, Sadeghi S, Tong MJ, Britten CD, Busuttil RW: Comparison of Triple-Drug Transcatheter Arterial Chemoembolization (TACE) With Single-Drug TACE Using Doxorubicin-Eluting Beads: Long-Term Survival in 313 Patients. AJR Am J Roentgenol 2017, 209(4):722-732. 
6. Lencioni R, Llovet JM: Modified RECIST (mRECIST) assessment for hepatocellular carcinoma. SEMIN LIVER DIS 2010, 30(1):52-60.

7. Villa E, Moles A, Ferretti I, Buttafoco P, Grottola A, Del BM, De Santis M, Manenti F: Natural history of inoperable hepatocellular carcinoma: estrogen receptors' status in the tumor is the strongest prognostic factor for survival. HEPATOLOGY 2000, 32(2):233-238.

8. Zhang Y, Fan W, Wang Y, Lu L, Fu S, Yang J, Huang Y, Yao W, Li J: Sorafenib With and Without Transarterial Chemoembolization for Advanced Hepatocellular Carcinoma With Main Portal Vein Tumor Thrombosis: A Retrospective Analysis. ONCOLOGIST 2015, 20(12):1417-1424.

9. Lu XJ, Dong J, Ji LJ, Luo JH, Cao HM, Xiao LX, Zhou J, Ling CQ: Safety and efficacy of TACE and gamma knife on hepatocellular carcinoma with portal vein invasion. GUT 2016, 65(4):715-716.

10. Long J, Zheng JS, Sun B, Lu N: Microwave ablation of hepatocellular carcinoma with portal vein tumor thrombosis after transarterial chemoembolization: a prospective study. HEPATOL INT 2016, 10(1):175-184.

11. Lu J, Zhang XP, Zhong BY, Lau WY, Madoff DC, Davidson JC, Qi X, Cheng SQ, Teng GJ: Management of patients with hepatocellular carcinoma and portal vein tumour thrombosis: comparing east and west. Lancet Gastroenterol Hepatol 2019, 4(9):721-730.

12. Luo J, Yan Z, Liu Q, Qu X, Wang J: Endovascular placement of iodine-125 seed strand and stent combined with chemoembolization for treatment of hepatocellular carcinoma with tumor thrombus in main portal vein. J VASC INTERV RADIOL 2011, 22(4):479-489.

13. Sidawy AN, Weiswasser JM, Waksman R: Peripheral vascular brachytherapy. J VASC SURG 2002, 35(5):1041-1047.

14. Lammer J, Malagari K, Vogl T, Pilleul F, Denys A, Watkinson A, Pitton M, Sergent G, Pfammatter T, Terraz S et al: Prospective randomized study of doxorubicin-eluting-bead embolization in the treatment of hepatocellular carcinoma: results of the PRECISION V study. Cardiovasc Intervent Radiol 2010, 33(1):41-52.

15. Kang YJ, Lee BC, Kim JK, Yim NY, Kim HO, Cho SB, Jeong YY: Conventional Versus Small Doxorubicin-eluting Bead Transcatheter Arterial Chemoembolization for Treating Barcelona Clinic Liver Cancer Stage 0/A Hepatocellular Carcinoma. Cardiovasc Intervent Radiol 2020, 43(1):55-64.

16. Brown KT, Do RK, Gonen M, Covey AM, Getrajdman GI, Sofocleous CT, Jarnagin WR, D'Angelica MI, Allen PJ, Erinjeri JP et al: Randomized Trial of Hepatic Artery Embolization for Hepatocellular Carcinoma Using Doxorubicin-Eluting Microspheres Compared With Embolization With Microspheres Alone. J CLIN ONCOL 2016, 34(17):2046-2053.

17. Karalli A, Teiler J, Haji M, Seth E, Brismar TB, Wahlin S, Axelsson R, Stal P: Comparison of lipiodol infusion and drug-eluting beads transarterial chemoembolization of hepatocellular carcinoma in a real-life setting. Scand J Gastroenterol 2019, 54(7):905-912.

18. Zhang ZH, Liu QX, Zhang W, Ma JQ, Wang JH, Luo JJ, Liu LX, Yan ZP: Combined endovascular brachytherapy, sorafenib, and transarterial chemobolization therapy for hepatocellular carcinoma patients with portal vein tumor thrombus. World J Gastroenterol 2017, 23(43):7735-7745 


\section{Figures}
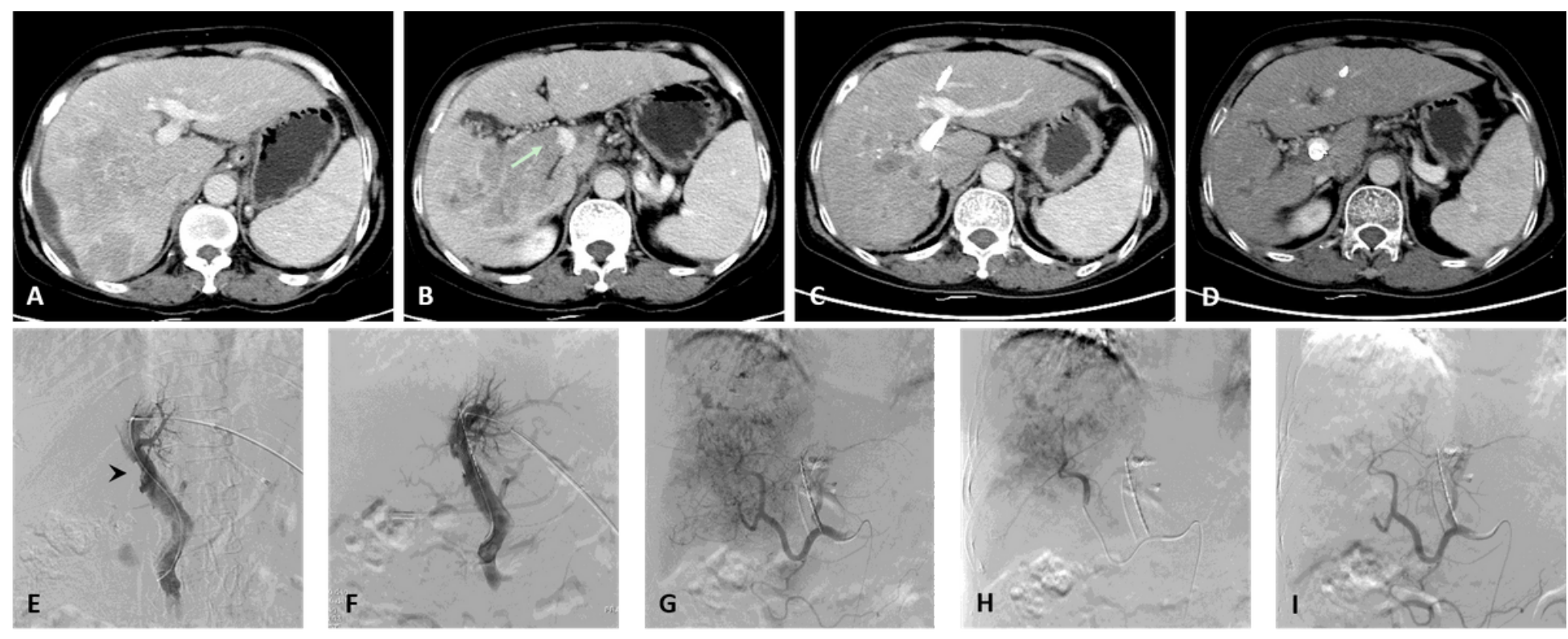

\section{Figure 1}

One case of the C-TACE group. The patient was a 60 years old female. Figure A: Preoperative CT images showed a huge tumor in the right lobe of the liver. Figure B: Tumor thrombus (arrow) was seen in the right branch and main portal vein. Figure C: After 3 months follow up, the tumor was significantly reduced. Figure D: The patency of portal vein stent was revealed after 3 months follow up. Figure E: Filling defect was showed in the main portal vein (arrow head) during venography. Figure F: Portal vein stent (diameter: $12 \mathrm{~mm}$, length: $60 \mathrm{~mm}$ ) was implanted and $12125 \mathrm{I}$ radioactive seeds were used. Panel G: Hepatic artery angiography showed large tumor staining of the right lobe of the liver. Figure $\mathrm{H}$ : Superselective embolization of the tumor artery branches with 50 milligrams of epirubicin mixed with $10 \mathrm{ml}$ lipiodol and 1 box of gelatin sponges was used to enhance embolization. Figure I: Angiography after embolization, tumor blood supply was significantly reduced.
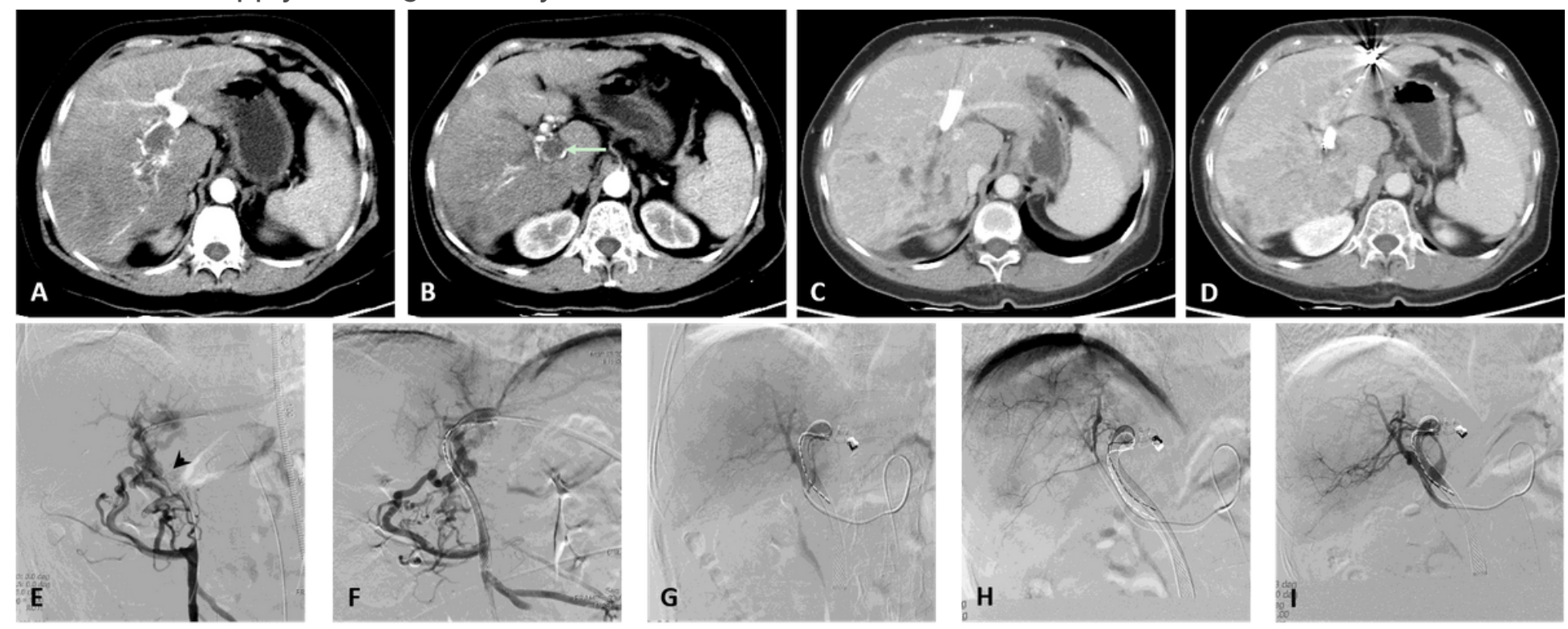
Figure 2

One case of the D-TACE group. The patient was a 69 years old female. Figure A: Preoperative CT images showed a huge tumor in the right lobe of the liver. Figure B: Tumor thrombus (arrow) was seen in the right branch and main portal vein. Figure C: After 3 months follow up, the tumor was significantly reduced.

Figure D: The patency of portal vein stent was revealed after 3 months follow up. Figure E: Filling defect was showed in the main portal vein (arrow head) during venography. Figure F: Portal vein stent (diameter: $10 \mathrm{~mm}$, length: $94 \mathrm{~mm}$ ) was implanted and $20125 \mathrm{I}$ radioactive seeds were used. Panel G: Hepatic artery angiography showed large tumor staining of the right lobe of the liver. Figure H: Superselective embolization of the tumor artery branches with 40 milligrams of epirubicin mixed with one bottle of DC beads. Figure I: Angiography after embolization, tumor blood supply was significantly reduced.

\section{Comparison of Liver Function}

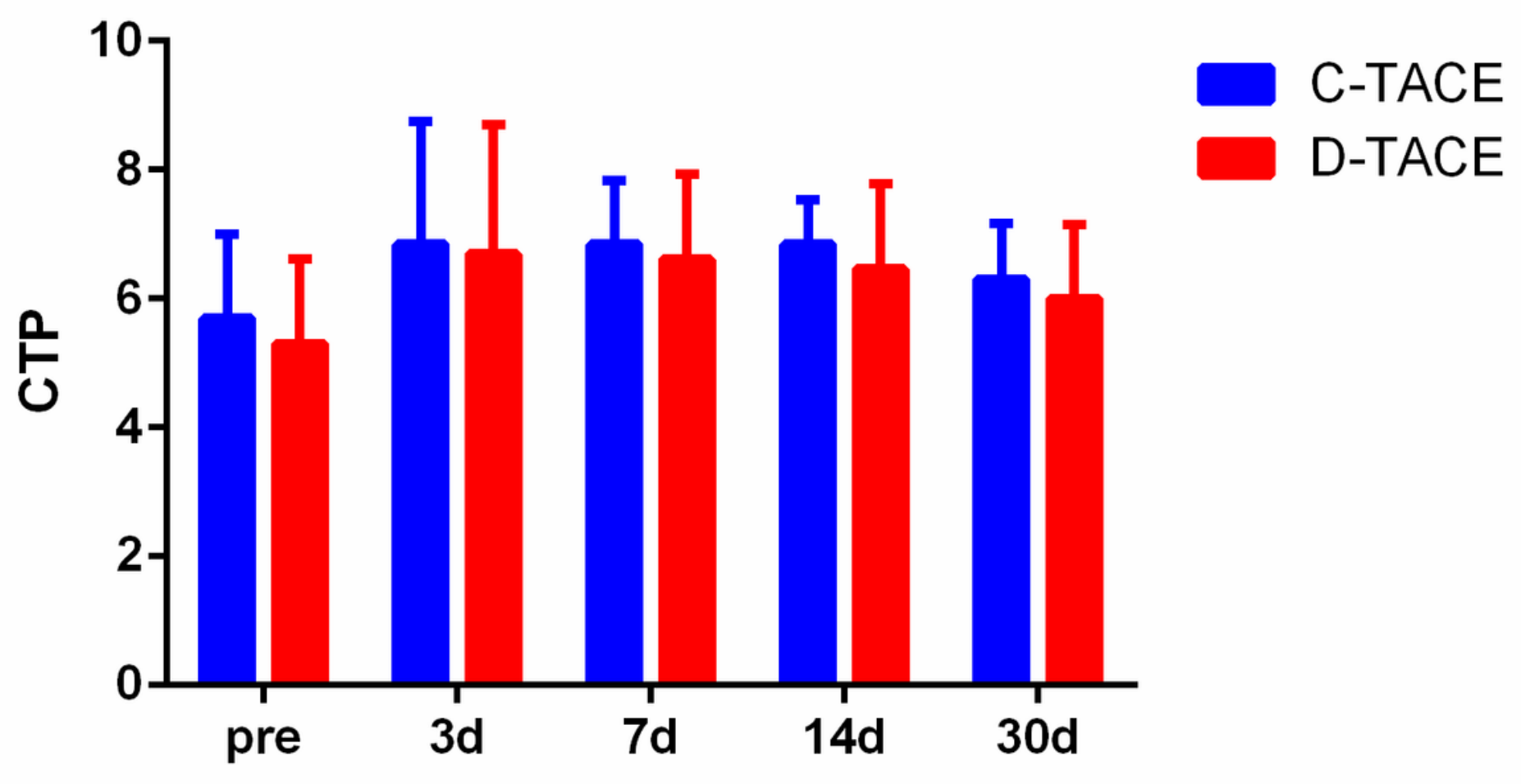

Figure 3

Comparison of liver function. There were no significant differences in Child-Pugh scores between the two groups before the procedures $(p=0.47)$ or at 3 days $(p=0.77), 7$ days $(p=0.66), 14$ days $(p=0.47)$, and 30 days $(p=0.56)$ after the procedures. 


\section{Comparison of PV Stent Patency Time}

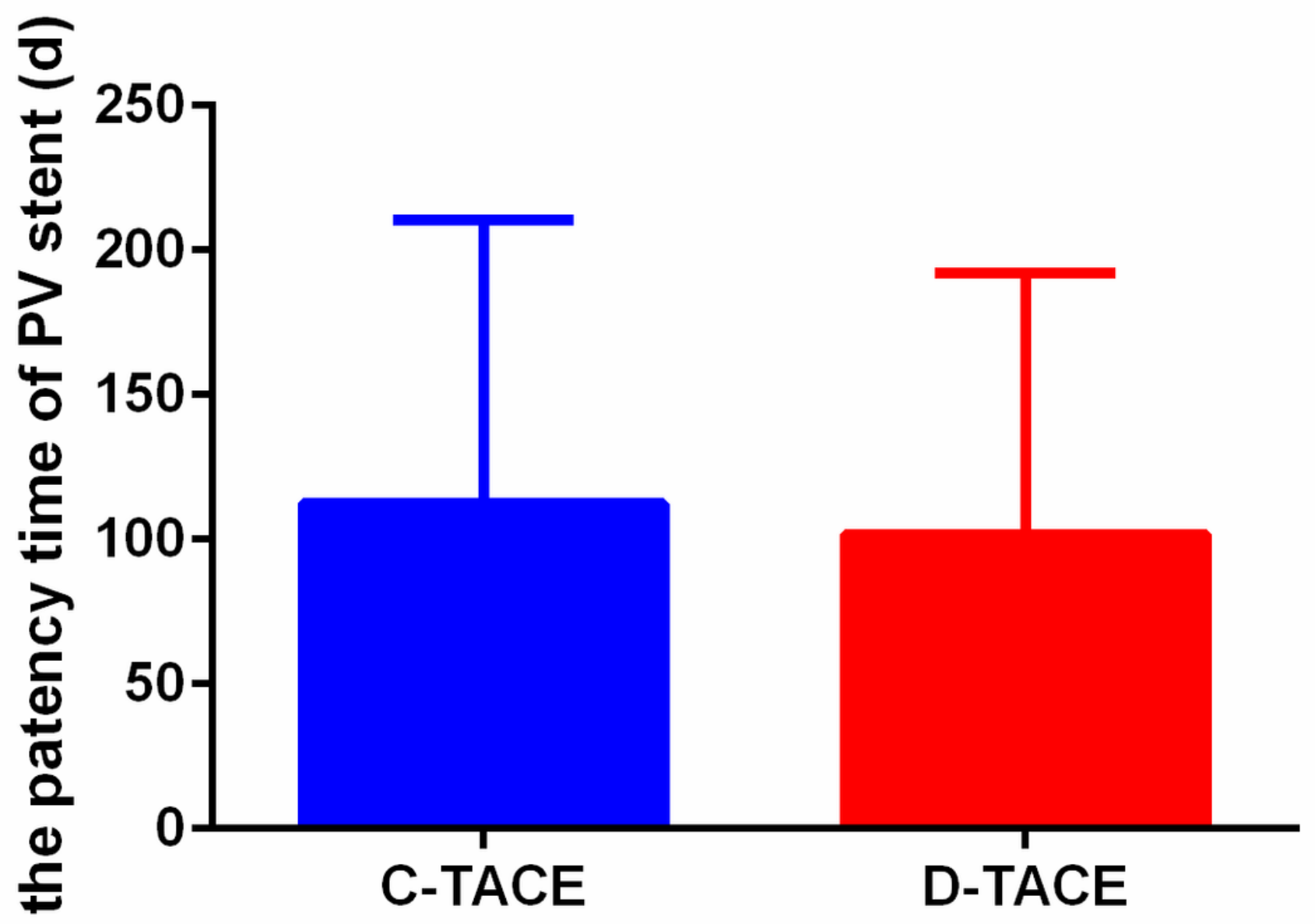

Figure 4

Comparison of PV stent patency time. The stent patency times of the two groups were $112.3 \pm 98.2$ days in the C-TACE group and 101.7 \pm 90.4 days in the D-TACE group, and there was no significant difference between the groups $(U=84, p>0.99)$.

PFS

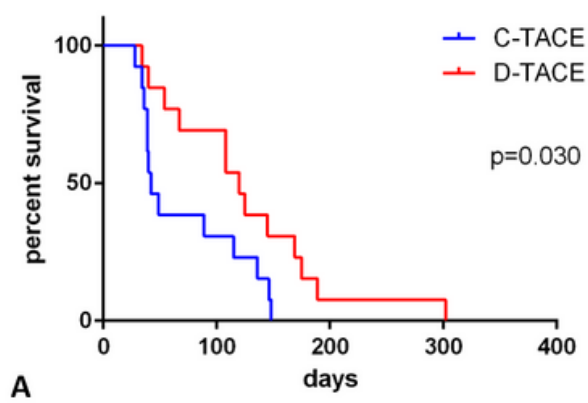

Survival Time from the Initial Diagnosis

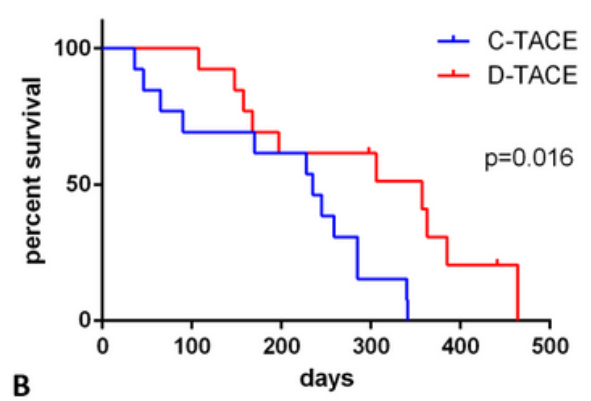

Survival Time from the First IR Therapy

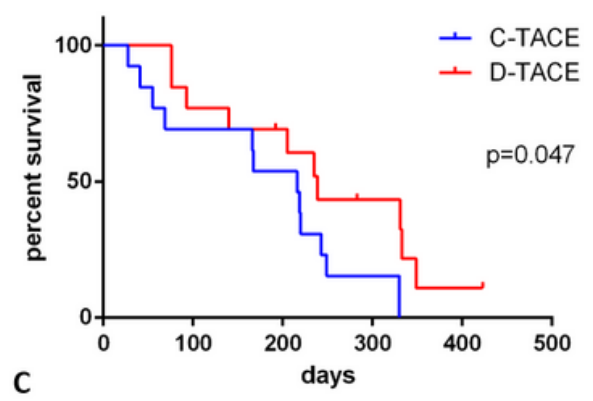

Figure 5 
Comparison of PFS and OS in the two groups. The median progression-free survival times of the two groups were 42 and 120 days, respectively $(p=0.030)$. From the initial diagnosis, the overall survival times of the two groups were 235 days and 357 days $(p=0.02)$. From the first interventional procedures, the overall survival times of the two groups were 216 days and 239 days, respectively $(p=0.047)$. 